\title{
PARAMETER ESTIMATION PROBLEMS FOR DISTRIBUTED SYSTEMS USING A MULTIGRID METHOD
}

\author{
BY \\ SHLOMO TA'ASAN AND PRAVIR DUTT \\ Institute for Computer Applications in Science and Engineering, \\ NASA Langley Research Center, Hampton, Virginia
}

\begin{abstract}
We consider the problem of estimating spatially varying coefficients of partial differential equations from observation of the solution and of the right-hand side of the equation. We assume that the observations are distributed in the domain and that enough observations are given. A method of discretization and an efficient multigrid method for solving the resulting discrete systems are described. Numerical results are presented for estimation of coefficients in an elliptic and a parabolic partial differential equation.
\end{abstract}

1. Introduction. This paper discusses the problem of estimating spatially varying coefficients of differential equations using observations of the solution and the right-hand side of the equation. We are restricting the discussion to distributed observations.

A common estimation procedure is to try to find parameters such that, by solving the equation, the solution is as close as possible (in some norm) to the observations [1] ("output least squares"). Another approach is to look at the equation as an equation for the parameters [2]. The approach we are discussing here is closer to the second one: we seek a solution (for the coefficients) in the least squares sense. This approach and the first approach are two special cases of another approach which estimates the parameters by minimizing a weighted sum of the error between observed and computed solutions with the residual of the equation. That is, given the equation

$$
L(g) V=f
$$

one identifies $g$ by minimizing over $(g, V) \in \mathscr{A}$ the functional

$$
J(V, g)=\left\|V-V^{*}\right\|^{2}+\sigma\left\|L(g) V-f^{*}\right\|^{2}
$$

where $V^{*}, f^{*}$ are the observed quantities, and $\mathscr{A}$ is a set of admissible pairs of functions.

Received August 5, 1989.

This research was supported under the National Aeronautics and Space Administration under NASA Contract Nos. NAS1-17070 and NASI-18107 while the authors were in residence at ICASE, NASA Langley Research Center, Hampton, VA 23665. 
Let $(\widetilde{v}(\sigma), \widetilde{g}(\sigma))$ be the minimum of $J$. Then $\sigma \rightarrow \infty,(\tilde{V}(\sigma), \tilde{g}(\sigma))$ converge to the solution of the "output least squares" solution. In the case $\sigma \rightarrow 0$, $(\widetilde{V}(\sigma), \widetilde{g}(\sigma))$ converge to the solution of the minimization problem discussed in this paper ("equation error approach").

The disadvantage of using the "equation error" approach is that one can treat only problems in which measurements are distributed in the domain and in which enough measurements are available. Also, this case may be more sensitive to noisy data than the "output least squares" approach because one applies $L(g)$ to $V^{*}$, and if $L(g)$ is a differential operator, a large error may be introduced even for a small amount of noise in $V^{*}$.

Section 2 describes the mathematical formulation of the problem and some basic rules for discretization. A multigrid procedure for solving the resulting equations is presented in Sec. 3. In Sec. 4, we present some numerical computations in which we estimate coefficients of an elliptic and parabolic equation. The elliptic case appears in problems involving large space structures [1], and the parabolic case arises in oil exploration and recovery [1].

2. Formulation of the problem and discretization method. Let $L(g)$ be a differential operator depending on a set of coefficients $\underline{g}(\underline{x})=\left(g_{1}(\underline{x}), \ldots, g_{l}(\underline{x})\right)$. We wish to estimate $\underline{g}$ from the observed solution $V(\underline{x})$ and a right-hand side of the equation

$$
L(\underline{g}) V(\underline{x})=f(\underline{x}) \quad \underline{x} \in \Omega \text {. }
$$

The problem is generally ill posed. In order that $\underline{g}(x)$ will be identifiable, $V(\underline{x})$ must satisfy some necessary conditions. (See [2] for example.) We assume throughout the paper that the observations $V(\underline{x}), f(\underline{x})$ are such that

(P1) observations are distributed in $\Omega$;

(P2) $\underline{V}(\underline{x}), f(\underline{x})$ are such that $\underline{g}(x)$ is identifiable;

(P3) $\underline{g}(\underline{x})$ depend continuously on $V(\underline{x}), f(\underline{x})$.

In this paper, we are not discussing conditions on $L(\underline{g}), V(\underline{x}), f(\underline{x})$ that imply (P2) and (P3). Basically, these assumptions imply that the identification problem is well posed. In a companion paper [5], we discuss such conditions for the example given in Sec. 4.1. Our approach for estimating $\underline{g}(\underline{x})$ will be to look for $\underline{g}(\underline{x})$ such that Eq. (2.1) will be satisfied in a least squares sense, i.e.,

$$
\min _{\underline{g}(\underline{x}) \in . \leftrightarrow}\left\|L(\underline{g}) V^{*}-f^{*}\right\|_{2}^{2}
$$

where $V^{*}, f^{*}$ are given at discrete points, and $\mathscr{A}$ is a class of admissible parameters. The first step is to construct an interpolant $\widetilde{V}^{*}$ that is defined everywhere in $\Omega$. This will define a problem on the continuous level. Next we consider the question of discretization.

Let the discrete domain be $\Omega^{h}$, on which $V_{*}^{h} f_{*}^{h}$ are given (by interpolation from the measurements). Let $\mathscr{A}^{h}, \mathscr{A}^{H}$ be spaces of discrete functions defined on the discrete domains $\Omega^{h}, \Omega^{H}$ respectively, where $\Omega^{H} \subseteq \Omega^{h}$. $\mathscr{A}^{H}$ will be the set of admissible parameters. The dimension of $\mathscr{A}^{H}$ may not be the same as that of $\mathscr{A}^{h}$. 
The discretization of $(2.1)$ is then

$$
L^{h}\left(g^{H}\right) V_{*}^{h}=f_{*}^{h} ; \quad g^{H} \in \mathscr{A}^{H}, V_{*}^{h}, f_{*}^{h} \in \mathscr{A}^{h}
$$

where $L^{h}\left(\underline{g}^{H}\right)$ is a matrix approximation to $L(\underline{g})$. The identification procedure then takes the form

$$
\min _{\underline{g}^{H} \in \mathscr{A}^{H}}\left\|L^{h}\left(g^{H}\right) V_{*}^{h}-f_{*}^{h}\right\|_{2}^{2} \text {. }
$$

The method one uses for discretization is a crucial one for the quality of the estimated quantities. We suggest the following rule:

Discretization Rule. The number of parameters in $\underline{g}^{H}$ should be less than the number of equations in (2.3).

The above rule is in accordance with results by others (for example [6]). This rule seems to guarantee that no spurious oscillations are developed in $\underline{g}^{H}$. In practice, we take $\underline{g}^{H}$ to be defined on a grid twice as coarse as the grid on which $V_{*}^{h}, f_{*}^{h}$ are defined.

Let the matrix $B\left(V^{h}\right)$ be defined by

$$
B\left(V^{h}\right) g^{H}=L^{h}\left(g^{H}\right) V^{h} .
$$

Note that, although $L^{h}\left(g^{H}\right)$ is a square matrix, $B\left(V^{h}\right)$ is a rectangular one since $\underline{g}^{H}$ involves less parameters than $V^{h}$. With the above definition of $B\left(V^{h}\right)$, our minimization problem takes the form

$$
\min _{g^{H} \in \mathscr{A}^{H}}\left\|B\left(V_{*}^{h}\right) g^{H}-f_{*}^{h}\right\|_{2} \text {. }
$$

This leads, in the case $B\left(V_{*}^{h}\right)$ is a linear operator, to the following system of equations

$$
\left[B\left(V_{*}^{h}\right)\right]^{T} B\left(V_{*}^{h}\right) \underline{g}^{H}=\left[B\left(V_{*}^{h}\right)\right]^{T} f_{*}^{h} .
$$

We next describe an efficient algorithm for solving the discrete system (2.7).

3. Multigrid procedure. The main element of a good multigrid procedure is a relaxation scheme. We start by describing this part.

Relaxation. Since $\left[B\left(V_{*}^{h}\right)\right]^{T} B\left(V_{*}^{h}\right)$ is symmetric positive definite, it seems that Gauss-Seidel relaxation will be appropriate. However, for this we have to compute $\left[B\left(V_{*}^{h}\right)\right]^{T} B\left(V_{*}^{h}\right)$. For general problems where $g^{H}, V_{*}^{h}$ lie on different grids, this may be too involved. As an alternative, we suggest Kaczmarz relaxation for

$$
B\left(V_{*}^{h}\right) g^{H}=f_{*}^{h} \text {. }
$$

From a theorem of Tanabe [3], this relaxation converges to the least squares solution plus the projection of the initial approximation onto the kernel of $\left[B\left(V_{*}^{h}\right)\right]^{T}$. Considering the task of programming the algorithm, it is much simpler to take this route over the one of computing $\left[B\left(V_{*}^{h}\right)\right]^{T} B\left(V_{*}^{h}\right)$.

The $i$ th step in Kaczmarz relaxation is given by For $j=1, \ldots, n$ do:

$$
g_{j}^{H} \leftarrow g_{j}^{H}+\delta_{i} b_{i j}
$$


where

$$
B\left(V_{*}^{h}\right)=\left(b_{i j}\right) \text { and } \delta_{i}=\frac{\left(f-B\left(V_{*}^{h}\right) g^{H}\right)_{i}}{\sum_{j}\left|b_{i j}\right|^{2}}
$$

We now come to the description of a multigrid cycle. We begin by describing a two-grid cycle.

Two-grid cycle. Suppose we are given two grids $\Omega^{h}, \Omega^{2 h}$ where $\Omega^{2 h} \subseteq \Omega^{h}$. Let $\mathscr{A}_{i}^{h}, \mathscr{A}_{i}{ }^{2 h}(i=1,2)$ be spaces of grid functions defined on $\Omega^{h}, \Omega^{2 h}$, respectively. Assume the $\Omega^{h}$-grid equations are

$$
B^{h} g^{h}=f^{h} \quad g^{h} \in \mathscr{A}_{1}^{h}, f^{h} \in \mathscr{A}_{2}^{h} .
$$

Let $I_{2 h}^{h}$ be an interpolation operator from $\mathscr{A}_{i}^{2 h}$ to $\mathscr{A}_{i}^{h}$, and $I_{h}^{2 h}=\left(I_{2 h}^{h}\right)^{*}$.

Given an approximate solution $\widetilde{g}^{h}$ to the above equation, a two-grid cycle for improving it consists of performing (A)-(D)

(A) Relax the equation $B^{h} g^{h}=f^{h} \nu_{1}$ times, starting with $\widetilde{g}^{h}$ yielding $\bar{g}^{h}$.

(B) Solve (approximately) the coarse grid equation

$$
B^{2 h} g^{2 h}=I_{h}^{2 h}\left(f^{h}-B^{h} \bar{g}^{h}\right) ; \quad B^{2 h}=I_{h}^{2 h} B^{h} I_{2 h}^{h},
$$

denote by $\widetilde{g}^{2 h}$ the (approximate) solution.

(C) Perform the correction

$$
\bar{g}^{h} \leftarrow \bar{g}^{h}+I_{2 h}^{h} \widetilde{g}^{2 h} .
$$

(D) Relax the equation $B^{h} g^{h}=f^{h} \nu_{2}$ times, starting with $\bar{g}^{h}$ yielding $\widetilde{g}^{h}$.

Note that $B^{2 h}$ is an operator from $\mathscr{A}_{1}^{2 h}$ to $\mathscr{A}_{2}^{2 h}$ and the definition given for it is only one possibility.

In order to obtain full efficiency of such an algorithm one solves the coarse grid equations in step (B) by a similar algorithm using a still coarser grid. Applying this idea recursively we get the basic multigrid cycle which is defined next.

Multigrid cycle. Given a sequence of discretizations with mesh sizes $h_{1}>h_{2}>$ $\cdots h_{m}$, on grids $\Omega^{k}$, where $h_{k}=2 h_{k+1}$ and $\Omega^{k-1} \subseteq \Omega^{k}$. Let the $h_{k}$-grid equation be

$$
B^{k} g^{k}=f^{k} ; \quad f^{k} \in \mathscr{A}_{2}^{k}, g^{k} \in \mathscr{A}_{1}^{k}
$$

where $B^{k}$ approximates $B^{k+1}(k<m)$ and $\mathscr{A}_{i}^{k}(i=1,2)$ are spaces of functions defined on the $h_{k}$-grid. Let $I_{k-1}^{k}$ be an interpolation operator from $\mathscr{A}_{i}^{k-1}$ to $\mathscr{A}_{i}^{k}$, and $I_{k}^{k-1}$ be a restriction operator from $\mathscr{A}_{i}^{k}$ to $\mathscr{A}_{i}^{k-1}$. The algorithm for improving a given approximate solution $\widetilde{g}^{k}$ to $(3.3)$ is denoted by

$$
\widetilde{g}^{k} \leftarrow M G\left(k, \tilde{g}^{k}, f^{k}\right)
$$

and is defined recursively as follows:

(1) If $k=1$, solve (3.3) by several relaxations otherwise do steps (A)-(D). 
(A) Perform $\nu_{1}$ relaxation sweeps on (3.3), resulting in a new approximation $\bar{g}^{k}$.

(B) Starting with $\widetilde{g}^{k-1}=0$, perform $\gamma$ times the following cycle

$$
\tilde{g}^{k-1} \leftarrow M G\left(k-1, \tilde{g}^{k-1}, I_{k}^{k-1}\left(f^{k}-B^{k} \bar{g}^{k}\right)\right),
$$

(C) Calculate

$$
\overline{\bar{g}}^{k} \leftarrow \bar{g}^{k}+I_{k-1}^{k} \widetilde{g}^{k-1} .
$$

(D) Perform $\nu_{2}$ additional relaxation steps on (3.3) starting with $\overline{\bar{g}}^{k}$ and yielding the final $\widetilde{g}^{k}$ of (3.4).

The cycle with $\gamma=1$ is called $V\left(\nu_{1}, \nu_{2}\right)$-cycle and the one with $\gamma=2$ is called $W\left(\nu_{1}, \nu_{2}\right)$.

Full Multigrid Algorithm (FMG). In order to obtain full efficiency, it is better to start from a good initial guess. This can be obtained by solving a similar problem on a coarser level. Applying the idea recursively, we get the following algorithm which is called Full Multigrid Algorithm and is denoted by $N$-FMG.

(1) Solve (3.3) for $k=1$ using several relaxations.

(2) $k=k+1$

$$
\widetilde{g}^{k}=\pi_{k-1}^{k} \widetilde{g}^{k-1}
$$

$\pi_{k-1}^{k}$ is an interpolation operator.

(3) Perform the cycle

$$
\tilde{g}^{k} \leftarrow M G\left(k, \widetilde{g}^{k}, f^{k}\right)
$$

$N$ times.

(4) If $k<m$, go to 2; otherwise, stop.

4. Numerical examples. In this section, we demonstrate the effectiveness of the method described. Results are given for two identification problems. In all cases the grids are finite and the spaces defined on them are of grid functions (finite dimensional), with the $l_{2}$-norm.

4.1. Elliptic case. Let $\underline{g}=g(x, y) ;(x, y) \in \Omega \equiv[0,1] \times[0,1]$, and

$$
L(\underline{g}) \equiv \frac{\partial}{\partial x} g \frac{\partial}{\partial x}+\frac{\partial}{\partial y} g \frac{\partial}{\partial y} .
$$

Determine $g(x, y)$ from observations of $V(x, y), f(x, y)$ which satisfy

$$
L(g) V=f \quad \text { in } \Omega .
$$

Discretization. $V, f$ were discretized on a uniform mesh

$$
\Omega^{h}=\{(i h, j h), 0 \leq i, j \leq N\} \quad N \cdot h=1 .
$$

$g$ was discretized on a mesh $\Omega^{H}$, with $H=2 h$. The discrete equation is

$$
\begin{aligned}
& g_{i+1 / 2, j}^{H}\left(\frac{U_{i+1, j}-U_{i, j}}{h^{2}}\right)-g_{i-1 / 2, j}^{H}\left(\frac{U_{i, j}-U_{i-1, j}}{h^{2}}\right) \\
& \quad+g_{i, j+1 / 2}^{H}\left(\frac{U_{i, j+1}-U_{i, j}}{h^{2}}\right)-g_{i, j-1 / 2}\left(\frac{U_{i, j}-U_{i, j-1}}{h^{2}}\right)=f_{i j}
\end{aligned}
$$


where $g_{i \pm 1 / 2, j}^{H}$ and $g_{i, j \pm 1 / 2}^{H}$ are given from the nodal values of $g^{H}$ on the grid $\Omega^{2 h}$ by linear interpolation. This gives approximately four times as many equations as there are unknowns. At every level of discretization, we maintained the relation between $g^{H}$, $U^{h}$, i.e., $g^{H}$ is always defined on a grid twice as coarse as the one for $U^{h}$. The $l_{2}$ norm of a function $U^{h}$ defined on a grid $\Omega^{h}$ is given by

$$
\left\|U^{h}\right\|_{2}=h \sqrt{\sum_{(i, j) \in \Omega^{h}}\left|U_{i j}^{h}\right|^{2}} .
$$

All interpolation was bilinear and residuals were transferred by the 9-point averaging operator.

$$
I_{k}^{k-1}=\frac{1}{16}\left[\begin{array}{lll}
1 & 2 & 1 \\
2 & 4 & 2 \\
1 & 2 & 1
\end{array}\right] \text {. }
$$

The following tables summarize the numerical experiments. In all the examples 1FMG using $W(2,1)$ cycle is shown. The numbers are taken at the end of the 1-FMG algorithm. In all the examples, the identification problem is well posed.

Example 1.

$$
\begin{aligned}
& V(x, y)=x(1-x) y(1-y) \\
& g(x, y)=1+x+y .
\end{aligned}
$$

Level \# $\left\|g^{h}-g^{*}\right\|_{2} \quad \|$ residuals $\|_{2}$

\begin{tabular}{|lll|}
\hline 1 & $.106(-4)$ & $.370(-5)$ \\
\hline 2 & $.328(-6)$ & $.190(-6)$ \\
\hline 3 & $.324(-7)$ & $.409(-7)$ \\
\hline 4 & .0 & $.0(-9)$ \\
\hline
\end{tabular}

Example 2.

$$
\begin{aligned}
& V(x, y)=\sin (\pi x) \sin (\pi y) \\
& g(x, y)=1+x+y .
\end{aligned}
$$

Level \# $\left\|g^{h}-g^{*}\right\|_{2} \quad \|$ residuals $\|_{2}$

\begin{tabular}{|lll|}
\hline 1 & .114 & $.452(-4)$ \\
\hline 2 & $.289(-1)$ & $.322(-1)$ \\
\hline 3 & $.694(-2)$ & $.818(-2)$ \\
\hline 4 & $.178(-2)$ & $.242(-2)$ \\
\hline
\end{tabular}


Example 3.

$$
\begin{aligned}
& V(x, y)=\sin (\pi x) \sin (\pi y) \\
& g(x, y)= \begin{cases}1+x, & x \leq .5 \\
1.5, & x \geq .5 .\end{cases}
\end{aligned}
$$

Level \# $\left\|g^{h}-g^{*}\right\|_{2} \quad \|$ residuals $\|_{2}$

\begin{tabular}{|lll|}
\hline 1 & $.812(-1)$ & $.289(-5)$ \\
\hline 2 & $.196(-1)$ & $.439(-1)$ \\
\hline 3 & $.530(-2)$ & $.181(-1)$ \\
\hline 4 & $.153(-2)$ & $.603(-2)$ \\
\hline
\end{tabular}

Example 4.

$$
\begin{aligned}
& V(x, y)=x(1-x) y(1-y) \\
& g(x, y)= \begin{cases}1+x, & x \leq .5 \\
1.5, & x \geq .5\end{cases}
\end{aligned}
$$

Level \# $\left\|g^{h}-g^{*}\right\|_{2} \quad \|$ residuals $\|_{2}$

\begin{tabular}{|lll|}
\hline 1 & $.206(-1)$ & $.237(-5)$ \\
\hline 2 & $.536(-2)$ & $.256(-2)$ \\
\hline 3 & $.204(-2)$ & $.959(-3)$ \\
\hline 4 & $.750(-3)$ & $.314(-4)$ \\
\hline
\end{tabular}

4.2. Parabolic case. Let $\underline{g}=(g(x, y), s(x, y)) ;(x, y) \in \Omega=[0,1]^{2}$; and

$$
L(g, s)=-s(x, y) \frac{\partial}{\partial t}+\frac{\partial}{\partial x} g \frac{\partial}{\partial x}+\frac{\partial}{\partial y} g \frac{\partial}{\partial y} .
$$

Determine $s(x, y), g(x, y)$ from observation of $V(x, y, t), f(x, y, t)$ such that the following equation holds

$$
L(g, s) V=f, \quad(x, y) \in \Omega, \quad 0 \leq t \leq T_{0} .
$$

In this problem, we used observed data at a few different times. We assumed that both $V$ and $V_{t}$ are given together with $f$.

Let $\Omega^{h}$ be defined as in the previous section and $\Gamma^{M}$ be defined as

$$
\Gamma^{M}=\left\{t_{n}: n=1, \ldots, M 0 \leq t_{n} \leq T_{0}\right\} .
$$

Discretization of $V(x, y, t), f(x, y, t)$, and $s(x, y, t)$ was done on $\Omega^{h} \times \Gamma^{M}$ while that of $g(x, y)$ used $\Omega^{H}(H=2 h)$. With such a discretization, the total number of unknowns is

$$
n^{2}+\frac{n^{2}}{4}
$$


The total number of equations is $n^{2}$ times the numbers of time observations. In our example, five time observations were used.

In defining coarse grids, we maintained the relation between $v, f, s, g$ as on the fine level, i.e., $v, f, s$ use a grid twice as fine as $g$ uses. We coarsened in space only, leaving the number of time measurements to be the same on all levels. Intergrid transfers were exactly as in the elliptic case (Sec. 4.1). The relaxation consists of two steps. The first one was a Kaczmarz relaxation in which both $g^{H}$ and $s^{h}$ were relaxed. The second one was a pointwise relaxation of $s^{h}$ only. In this relaxation, the points $(i, j) \in \Omega^{h}$ were scanned in lexicographic ordering, and at each point $s_{i j}^{h}$ was changed to minimize

$$
\left\|L^{h}\left(g^{H}, s^{h}\right) V_{*}^{h}-f^{h}\right\| .
$$

The following tables summarize the numerical results. Results are given for 3FMG using $W(2,2)$ cycle.

Example 1.

$$
\begin{aligned}
& v(x, y, t)=\sin (\pi x) \sin (\pi y)(t+1) \\
& g(x, y)=1+x+y \\
& s(x, y)=1
\end{aligned}
$$

Level \# Cycle \# $\left\|g^{h}-g^{*}\right\| \quad\left\|s^{h}-s^{*}\right\| \quad \|$ residuals $\|_{2}$

\begin{tabular}{|lllll|}
\hline 1 & 10 & .114 & $8.11(-5)$ & $3.52(-5)$ \\
\hline 2 & 1 & $2.75(-2)$ & $1.17(-2)$ & $4.64(-3)$ \\
\hline & 2 & $2.75(-2)$ & $5.06(-3)$ & $4.14(-3)$ \\
\hline & 3 & $2.75(-2)$ & $2.72(-3)$ & $3.81(-3)$ \\
\hline & 4 & $2.75(-2)$ & $1.98(-3)$ & $3.54(-3)$ \\
\hline 3 & 1 & $6.81(-3)$ & $1.96(-2)$ & $1.50(-3)$ \\
\hline & 2 & $6.79(-3)$ & $8.75(-3)$ & $1.38(-3)$ \\
\hline & 3 & $6.78(-3)$ & $3.95(-3)$ & $1.29(-3)$ \\
\hline & 4 & $6.78(-3)$ & $1.95(-3)$ & $1.20(-3)$ \\
\hline 4 & 1 & $1.77(-3)$ & $3.30(-2)$ & $2.41(-3)$ \\
\hline & 2 & $1.72(-3)$ & $1.98(-2)$ & $2.58(-3)$ \\
\hline 3 & $1.70(-3)$ & $1.20(-2)$ & $2.67(-3)$ \\
\hline 4 & $1.69(-3)$ & $7.96(-3)$ & $2.56(-3)$ \\
\hline
\end{tabular}

Example 2.

$$
\begin{aligned}
& V(x, y, t)=\sin (\pi x) \sin (\pi y)(t+1), \quad s(x, y)=1 \\
& g(x, y)= \begin{cases}1+x & x \leq .5 \\
1.5 & x \geq .5\end{cases}
\end{aligned}
$$


Level \# Cycle \# $\left\|g^{h}-g^{*}\right\| \quad\left\|s^{h}-s^{*}\right\| \quad \|$ residuals $\|_{2}$

\begin{tabular}{|lllll|}
\hline 1 & 10 & $8.19(-2)$ & $4.49(-5)$ & $1.93(-5)$ \\
\hline 2 & 1 & $1.90(-2)$ & $6.98(-3)$ & .137 \\
\hline & 2 & $1.90(-2)$ & $1.01(-2)$ & .128 \\
\hline 3 & $1.91(-2)$ & $1.46(-2)$ & .119 \\
\hline & 4 & $1.92(-2)$ & $1.88(-2)$ & .111 \\
\hline 3 & 1 & $5.08(-3)$ & $1.02(-2)$ & $5.78(-2)$ \\
\hline & 2 & $5.17(-3)$ & $7.02(-3)$ & $5.37(-2)$ \\
\hline 3 & $5.22(-3)$ & $6.86(-3)$ & $4.99(-2)$ \\
\hline 4 & $5.25(-3)$ & $7.79(-3)$ & $4.66(-2)$ \\
\hline 4 & 1 & $1.43(-3)$ & $1.68(-2)$ & $2.29(-2)$ \\
\hline & 2 & $1.44(-3)$ & $1.01(-2)$ & $2.17(-2)$ \\
\hline 3 & $1.45(-3)$ & $6.83(-3)$ & $2.06(-2)$ \\
\hline 4 & $1.46(-3)$ & $5.59(-3)$ & $1.97(-2)$ \\
\hline
\end{tabular}

4.3. Discussion. These numerical examples clearly demonstrate the effectiveness of the method of discretization as well as the solution process.

In the elliptic problem, the coefficients converge in a rate of $O\left(h^{2}\right)$ in the smooth case. When derivatives of the coefficients are not smooth, a slower rate is obtained as one would expect. The multigrid algorithm solves the problem up to the level of discretization errors in just one multigrid cycle of the type FMG- $W(2,1)$.

In the parabolic case, the coefficient $g(x, y)$ shows $O\left(h^{2}\right)$ convergence (in the smooth case), and this is obtained after the first cycle. $s(x, y)$ behaves worse, taking more cycles to reach the level of discretization error. This can be explained as follows: a change of order $O\left(h^{2}\right)$ in $g(x, y)$ may lead to a change of $O(h)$ in $s(x, y)$. Hence $s(x, y)$ may reach convergence only after $g(x, y)$ has converged. It is possible that, although $s(x, y)$ has not reached convergence, $g(x, y)$ will be accurate up to discretization errors if the error in $s(x, y)$ times $V_{t}(x, y, t)$ is of the level of truncation errors. The behavior of the residuals in examples of $\S 4.2$ is not clear. It goes up when going from level three to level four, and it seems that it should have gone down.

\section{REFERENCES}

[1] H. T. Banks, A survey of some problems and recent results for parameter estimation and optimal control in delay and distributed parameter systems, ICASE Report No. 81-26

[2] G. R. Richter, An inverse problem for the steady state diffusion equation, SIAM J. Appl. Math. 41 (2), 1981

[3] K. Tanabe, Projection methods for solving a singular system of linear equations and its applications, Numer. Math. 17, 203-214 (1971) 
[4] A. Brandt, Multigrid Techniques: 1984 Guide with Applications to Fluid Dynamics, Monograph available as GMD-Study, no. 85, from GMD-F1T, Postfach 1240, D-5205, St. Augustin 1, W. Germany

[5] P. Dutt and S. Ta' asan, A spline-based parameter estimation technique for static models of elastic structures, Appl. Numer. Math. 5, 161-175 (1989)

[6] H. T. Banks and D. W. Iles, On compactness of admissible parameter sets: Convergence and stability in inverse problems for distributed systems, ICASE Report No. 86-38, NASA CR-178128, 1986 